\title{
THE LEAST EIGENVALUE OF THE GRAPHS WHOSE COMPLEMENTS ARE CONNECTED AND HAVE PENDENT PATHS
}

\author{
Chen Wang ${ }^{1}$, Guidong $\mathrm{Yu}^{2}$, Wei $\mathrm{Sun}^{2}$, Jinde $\mathrm{Cao}^{3}$ \\ ${ }^{1}$ School of Mathematics and Statistics, Guizhou University, Guiyang 550025, China \\ ${ }^{2}$ School of Mathematics and Computation Sciences, Anqing Normal University, \\ Anqing 246133, China \\ ${ }^{3}$ School of Mathematics, Southeast University, Nanjing, Jiangsu 210096, China
}

Submitted: 27th January 2018; Accepted: 16th March 2018

\begin{abstract}
The adjacency matrix of a graph is a matrix which represents adjacent relation between the vertices of the graph. Its minimum eigenvalue is defined as the least eigenvalue of the graph. Let $G_{n}$ be the set of the graphs of order $n$, whose complements are connected and have pendent paths. This paper investigates the least eigenvalue of the graphs and characterizes the unique graph which has the minimum least eigenvalue in $G_{n}$.
\end{abstract}

Keywords: graph, complement, pendent path, adjacency matrix, the least eigenvalue

\section{Introduction}

Let $G:=(V(G), E(G))$ be a simple graph of order $n$, where $V(G)=\left\{v_{1}, v_{2}, \cdots, v_{n}\right\}$ be vertex set, $E(G)=\left\{e_{1}, e_{2}, \cdots, e_{m}\right\}$ be the edge set. The set of neighbours of a vertex $v_{i} \in V(G)$ be denoted by $N_{G}\left(v_{i}\right)=\left\{v_{j}: v_{j} v_{i} \in E(G)\right\}$. If a path $P=v_{0} e_{1} v_{1} e_{2} \cdots e_{k} v_{k}$ satisfies $d_{G}\left(v_{0}\right) \geq 3, d_{G}\left(v_{i}\right)=$ $2, i=1,2, \cdots, k-1, d\left(v_{k}\right)=1$ and $k \geq 2, P$ is called a pendent path of length $k$, where $d_{G}\left(v_{i}\right)=1$ $N_{G}\left(v_{i}\right) \mid$. For any $v_{i}, v_{j} \in V(G)$, if there is a path such that $v_{i}$ and $v_{j}$ are its ends, $G$ is called a connected graph. The complement of $G$ is denoted by $G^{c}:=\left(V(G), E^{c}(G)\right)$, where $E^{c}(G):=\left\{v_{i} v_{j}\right.$ : $\left.v_{i}, v_{j} \in V(G), v_{i} \neq v_{j}, v_{i} v_{j} \notin E(G)\right\}$.

The degree matrix $D(G)$ of $G$ is diagonal square matrix of order $n$, where $d_{i j}=0$ when $i \neq j, d_{i i}=$ $d_{G}\left(v_{i}\right), d_{i j}$ be $i j$-entry of $D(G)$. The adjacency matrix $A(G)$ of $G$ is the square matrix of order $n$, where $a_{i j}=1$ when $v_{i} v_{j} \in E(G), a_{i j}=0$ when $v_{i} v_{j} \notin E(G), a_{i j}$ be the $i j-$ entry of $A(G)$. The signless Laplacian matrix of $G$ is defined to be $Q(G)=D(G)+A(G)$. In addition, the Laplacian matrix of $G$ is defined by $L(G)=D(G)-A(G)$. Since $A(G), Q(G), L(G)$ are real symmetric matrix, their eigenvalues are real numbers and can be arranged. Let the eigenvalues of $A(G)$ arrange as $: \lambda_{1}(G) \geq \lambda_{2}(G) \geq \cdots \geq \lambda_{n}(G)$. The largest eigenvalue $\lambda_{1}(G)$ of $A(G)$ is called the spectral radius of $G$. The minimum eigenvalue $\lambda_{n}(G)$ of $A(G)$, simply denoted by $\lambda_{\min }(G)$, is called the least eigenvalue of $G$. The corresponding unit eigenvectors of $\lambda_{\min }(G)$ are called the first eigenvectors of $G$. Similarly, the largest eigenvalue of $Q(G)$ be defined as the signless Laplacian spectral radius of graph $G$, and the minimum eigenvalue of $Q(G)$ be defined as the least signless Laplacian eigenvalue of graph $G$.

There are many research results with respect to the (signless Laplacian) spectral radius of graphs. However, relative to the (signless Laplacian) spec- 
tral radius, there are few results on the (signless Laplacian) least eigenvalue. Especially, when the structures of graphs are very complex, but the structures of their complements are simple, we naturally think whether we can study the (signless Laplacian) minimum eigenvalue of the graphs from structure of their complements; see[1-16]. In this paper, we also study the least eigenvalue of graphs from their complements. Let $G_{n}$ be the set of the graphs of order $n$, whose complements are connected and have pendent paths. This paper investigates the least eigenvalue of the graphs and characterizes the unique graph which has the minimum least eigenvalue in $G_{n}$.

\section{Preliminaries}

In the following, we will introduce some definitions. Let $G$ be a graph of order $n$, a vector $\mathbf{x} \in R^{n}$ is defined on $G$.

One can find that, for an arbitrary vector $\mathbf{x} \in R^{n}$,

$$
\mathbf{x}^{T} A(G) \mathbf{x}=2 \sum_{u v \in E(G)} x_{u} x_{v},
$$

and if $\mathbf{x}(\neq 0)$ is an eigenvector of $A(G)$, which is correspond to the eigenvalue $\lambda$ of $A(G)$, then

$$
\lambda x_{v}=\sum_{u \in N_{G}(v)} x_{u}, \text { for each } v \in V(G)
$$

if $\mathbf{x}$ is unit vector, then

$$
\lambda_{\min }(G) \leq \mathbf{x}^{T} A(G) \mathbf{x},
$$

with equality when and only when $\mathbf{x}$ is the first eigenvector of $G$.

We can also find that $A\left(G^{c}\right)=J-I-A(G)$, where $J, I$ denote the all-one square matrix and the identity matrix of order $n$, respectively. Then for an arbitrary vector $\mathbf{x} \in R^{n}$,

$$
\mathbf{x}^{T} A\left(G^{C}\right) \mathbf{x}=\mathbf{x}^{T}(J-I) \mathbf{x}-\mathbf{x}^{T} A(G) \mathbf{x} .
$$

We introduce a special graph $G(p, q)$ of order $n=p+q+2(p \geq 0, q \geq 1)$, which is obtained from two disjoint complete graphs $K_{p}, K_{q}$, and edge $v_{4} v_{5}$ by joining one vertex $v_{1}$ of $K_{p}$ and one vertex $v_{2}$ of $K_{q}$, and joining one vertex $v_{3}$ of $K_{q}$ and $v_{4}$; see Figure 1. In particular, when $q=1, v_{2}=v_{3}$; when $p=0, G(0, q)$ is obtained from complete graph $K_{q}$ and edge $v_{4} v_{5}$ by joining one vertex $v_{3}$ of $K_{q}$ and $v_{4}$.

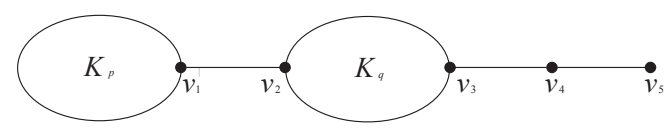

Figure 1. The graph $G(p, q)$

Lemma 2.1 Let $A$ be a real symmetric square matrix of order $n, B$ be the principal submatrix of $A$ of order $m$, and $\mu_{1}(A) \geq \mu_{2}(A) \geq \cdots \geq \mu_{n}(A), \mu_{1}(B) \geq$ $\mu_{2}(B) \geq \cdots \geq \mu_{m}(B)$ are respectively the eigenvalues of $A$ and $B$, then $\mu_{n-m+i}(A) \leq \mu_{i}(B) \leq \mu_{i}(A)$ for $i=1,2, \cdots, m$.

Lemma 2.2 Given a positive integer $n(n \geq 21)$, if $n=p+q+2, p, q$ be integer, $p \geq 0, q \geq 1$, then

$$
\lambda_{\text {min }}\left(G(p, q)^{c}\right) \geq \lambda_{\text {min }}\left(G(\lceil n / 2\rceil-1,\lfloor n / 2\rfloor-1)^{c}\right),
$$

with equality when and only when $p=\lceil n / 2\rceil-$ $1, q=\lfloor n / 2\rfloor-1$.

Proof: Let $G(p, q)$ be as shown in Fig. 2.1. Because $K_{2} \subset G(p, q)^{c}, \lambda_{\text {min }}\left(K_{2}\right)=-1$, and according to Lemma 2.1, we have

$$
\lambda_{\min }\left(G(p, q)^{c}\right) \leq-1 .
$$

Let $\mathbf{x}$ be a first eigenvector of $G(p, q)^{c}$.

Case 1: $p=0$. By equations (2.2) and (2.5), all the vertices in $V\left(K_{q}\right)$ except $v_{3}$ have the same values, which are given by $\mathbf{x}$, denoted by $x_{1}$. Denote $x_{v_{3}}=x_{2}, x_{v_{4}}=x_{3}, x_{v_{5}}=x_{4}$, and let $\lambda_{\min }(G(0, n-$ $\left.2)^{c}\right)=\lambda$. Also by equation(2.2), we obtain

$$
\left\{\begin{array}{l}
\lambda x_{1}=x_{3}+x_{4} \\
\lambda x_{2}=x_{4} \\
\lambda x_{3}=(n-3) x_{1} \\
\lambda x_{4}=(n-3) x_{1}+x_{2} .
\end{array}\right.
$$

The above equations are transformed into a matrix equation $(B-\lambda I) \mathbf{x}^{\prime}=0$, where $\mathbf{x}^{\prime}=\left(x_{1}, x_{2}, x_{3}, x_{4}\right)^{T}$,

$$
B=\left(\begin{array}{cccc}
0 & 0 & 1 & 1 \\
0 & 0 & 0 & 1 \\
n-3 & 0 & 0 & 0 \\
n-3 & 1 & 0 & 0
\end{array}\right)
$$

We have

$$
f_{1}(x)=\operatorname{det}(B-x I)=x^{4}-(2 n-5) x^{2}+n-3,
$$

then $\lambda$ is the smallest root of $f_{1}(x)=0$, therefore

$\lambda=-\sqrt{\left(2 n-5+\sqrt{4 n^{2}-24 n+37}\right) / 2}>-\sqrt{2 n-5}$. 
Case 2: $p=1$. By equations (2.2) and (2.5), all the vertices in $V\left(K_{q}\right)$ except $v_{2}, v_{3}$ have the same values, which are given by $\mathbf{x}$, denoted by $x_{1}$. Denote $x_{v_{1}}=x_{2}, x_{v_{2}}=x_{3}, x_{v_{3}}=x_{4}, x_{v_{4}}=x_{5}, x_{v_{5}}=x_{6}$, and $\lambda_{\min }\left(G(1, n-3)^{c}\right)=\lambda$. Also by equation(2.2), we have

$$
\left\{\begin{array}{l}
\lambda x_{1}=x_{2}+x_{5}+x_{6} \\
\lambda x_{2}=(n-5) x_{1}+x_{4}+x_{5}+x_{6}, \\
\lambda x_{3}=x_{5}+x_{6} \\
\lambda x_{4}=x_{2}+x_{6} \\
\lambda x_{5}=(n-5) x_{1}+x_{2}+x_{3} \\
\lambda x_{6}=(n-5) x_{1}+x_{2}+x_{3}+x_{4} .
\end{array}\right.
$$

The above equations are transformed into a matrix equation $(B-\lambda I) \mathbf{x}^{\prime}=0$, where $\mathbf{x}^{\prime}=\left(x_{1}, x_{2}, x_{3}\right.$, $\left.x_{4}, x_{5}, x_{6}\right)^{T}$,

$$
B=\left(\begin{array}{cccccc}
0 & 1 & 0 & 0 & 1 & 1 \\
n-5 & 0 & 0 & 1 & 1 & 1 \\
0 & 0 & 0 & 0 & 1 & 1 \\
0 & 1 & 0 & 0 & 0 & 1 \\
n-5 & 1 & 1 & 0 & 0 & 0 \\
n-5 & 1 & 1 & 1 & 0 & 0
\end{array}\right)
$$

We have $f_{2}(x)=\operatorname{det}(B-x I)=x^{6}-(3 n-9) x^{4}-$ $(4 n-18) x^{3}+(4 n-16) x^{2}+2(n-5) x-(n-5)$. Because $n \geq 21$, we have

$$
\begin{aligned}
f_{2}(-\sqrt{2 n-5})= & (2 n-5)\left(-2 n^{2}+17 n-36\right) \\
& +\sqrt{2 n-5}\left(8 n^{2}-58 n+100\right) \\
& -(n-5) \\
< & 0,
\end{aligned}
$$

this implies $\lambda_{\min }\left(G(1, n-3)^{c}\right)<-\sqrt{2 n-5}$.

Case 3: $q=1$. By equations (2.2) and (2.5), all the vertices in $V\left(K_{p}\right)$ except $v_{1}$ have the same values, which are given by $\mathbf{x}$, denoted by $x_{1}$. Denote $x_{v_{1}}=x_{2}, x_{v_{2}\left(v_{3}\right)}=x_{3}, x_{v_{4}}=x_{4}, x_{v_{5}}=x_{5}$, and $\lambda_{\min }\left(G(n-3,1)^{c}\right)=\lambda$. Also by equation(2.2), we have

$$
\left\{\begin{array}{l}
\lambda x_{1}=x_{3}+x_{4}+x_{5}, \\
\lambda x_{2}=x_{4}+x_{5}, \\
\lambda x_{3}=(n-4) x_{1}+x_{5}, \\
\lambda x_{4}=(n-4) x_{1}+x_{2}, \\
\lambda x_{5}=(n-4) x_{1}+x_{2}+x_{3} .
\end{array}\right.
$$

The above equations are transformed into a matrix equation $(B-\lambda I) \mathbf{x}^{\prime}=0$, where $\mathbf{x}^{\prime}=\left(x_{1}, x_{2}, x_{3}\right.$, $\left.x_{4}, x_{5}\right)^{T}$,

$$
B=\left(\begin{array}{ccccc}
0 & 0 & 1 & 1 & 1 \\
0 & 0 & 0 & 1 & 1 \\
n-4 & 0 & 0 & 0 & 1 \\
n-4 & 1 & 0 & 0 & 0 \\
n-4 & 1 & 1 & 0 & 0
\end{array}\right)
$$

We have $f_{3}(x)=\operatorname{det}(B-x I)=-x^{5}+(3 n-$ $9) x^{3}+2(n-4) x^{2}-(3 n-11) x$. When $n \geq 21$ and $x<-1$, we have $(-x) f_{3}(x)-f_{2}(x)=(n-$ $5)\left(2 x^{3}-x^{2}-2 x+1\right)<0$. Then by $\lambda_{\min }(G(1, n-$ $\left.3)^{c}\right)<-\sqrt{2 n-5}$, we have $\lambda_{\text {min }}\left(G(n-3,1)^{c}\right)<$ $\lambda_{\min }\left(G(1, n-3)^{c}\right)$.

Case 4: $p, q \geq 2$. By equations (2.2) and (2.5), all the vertices in $V\left(K_{p}\right)$ except $v_{1}$ have the same values, which are given by $\mathbf{x}$, denoted by $x_{1}$, all the vertices in $V\left(K_{q}\right)$ except $v_{2}, v_{3}$ have the same values given by $\mathbf{x}$, say $x_{4}$. Denote $x_{v_{1}}=x_{2}, x_{v_{2}}=x_{3}, x_{v_{3}}=$ $x_{5}, x_{v_{4}}=x_{6}, x_{v_{5}}=x_{7}$, and $\lambda_{\min }\left(G(p, q)^{c}\right)=\lambda$. Also by equation(2.2), we have

$$
\left\{\begin{array}{l}
\lambda x_{1}=x_{3}+(q-2) x_{4}+x_{5}+x_{6}+x_{7}, \\
\lambda x_{2}=(q-2) x_{4}+x_{5}+x_{6}+x_{7}, \\
\lambda x_{3}=(p-1) x_{1}+x_{6}+x_{7}, \\
\lambda x_{4}=(p-1) x_{1}+x_{2}+x_{6}+x_{7}, \\
\lambda x_{5}=(p-1) x_{1}+x_{2}+x_{7}, \\
\lambda x_{6}=(p-1) x_{1}+x_{2}+x_{3}+(q-2) x_{4}, \\
\lambda x_{7}=(p-1) x_{1}+x_{2}+x_{3}+(q-2) x_{4}+x_{5} .
\end{array}\right.
$$

The above equations are transformed into a matrix equation $(B-\lambda I) \mathbf{x}^{\prime}=0$, where $\mathbf{x}^{\prime}=$ $\left(x_{1}, x_{2}, x_{3}, x_{4}, x_{5}, x_{6}, x_{7}\right)^{T}$,

$$
B=\left(\begin{array}{ccccccc}
0 & 0 & 1 & q-2 & 1 & 1 & 1 \\
0 & 0 & 0 & q-2 & 1 & 1 & 1 \\
p-1 & 0 & 0 & 0 & 0 & 1 & 1 \\
p-1 & 1 & 0 & 0 & 0 & 1 & 1 \\
p-1 & 1 & 0 & 0 & 0 & 0 & 1 \\
p-1 & 1 & 1 & q-2 & 0 & 0 & 0 \\
p-1 & 1 & 1 & q-2 & 1 & 0 & 0
\end{array}\right)
$$

We have

$$
\begin{aligned}
& f_{4}(x ; p, q)=\operatorname{det}(B-x I)= \\
& -x^{7}+(p q+2 n-6) x^{5}+(4 p q-2 p-4) x^{4} \\
& -(2 p q+q+n-7) x^{3}-(6 p q-4 n-4 p+12) x^{2} \\
& +(p q-p-1) x+2(p-1)(q-2) .
\end{aligned}
$$

Since $h(x)=x(n-2-x)$ takes the minimum value $h(2)=2(n-4)$ when $2 \leq x \leq n-4$. So 
$p q=p(n-2-p) \geq 2(n-4)$ when $p, q \geq 2$ and $n=$ $p+q+2$. Then $f(-7 ; p, q)=911121-32734 n-$ $4944 p-6816 p q<0$ when $p, q \geq 2$ and $n=p+q+$ $2 \geq 21$, which implies $\lambda<-7$.

Case 4.1: $p \geq q+2$. When $x<-7$, we have

$$
\begin{aligned}
& f_{4}(x ; p, q)-f_{4}(x ; p-1, q+1) \\
= & (q-p+1) x^{5}-(4 p-4 q-2) x^{4}+(2 p-2 q-1) x^{3} \\
& +(6 p-6 q-2) x^{2}-(p-q) x-(2 p-2 q) \\
> & -x^{3}\left((p-q-1) x^{2}+(4 p-4 q-2) x\right. \\
& -(2 p-2 q-1)) \\
> & 0 .
\end{aligned}
$$

Since $\lambda$ is the smallest root of $f_{4}(x ; p, q)=0$, i.e. $f_{4}(\lambda ; p, q)=0$, then $f_{4}(\lambda ; p-1, q+1)<0$, this implies $\lambda_{\text {min }}\left(G(p, q)^{c}\right)>\lambda_{\text {min }}\left(G(p-1, q+1)^{c}\right)$. So $\lambda_{\min }\left(G(n-4,2)^{c}\right)>\lambda_{\text {min }}\left(G(n-5,3)^{c}\right)>\cdots>$ $\lambda_{\min }\left(G(\lceil n / 2\rceil-1,\lfloor n / 2\rfloor-1)^{c}\right)$.

Case 4.2: $q \geq p+1$. When $x<-7$ and $q \geq$ $p+2$, we have

$$
\begin{aligned}
& f_{4}(x ; p, q)-f_{4}(x ; p+1, q-1) \\
= & -(q-p-1) x^{5}-(4 q-4 p-6) x^{4}+(2 q-2 p-3) x^{3} \\
& +(6 q-6 p-10) x^{2}-(q-p-2) x-(2 q-2 p-4) \\
> & -x^{3}\left((q-p-1) x^{2}+(4 q-4 p-6) x-(2 q-2 p-3)\right)
\end{aligned}
$$$$
>0 \text {. }
$$

When $x<-7$ and $q=p+1$, we have $f_{4}(x ; p, q)-f_{4}(x ; p+1, q-1)=2 x^{4}-x^{3}-4 x^{2}+$ $x+2>0$.

Since $\lambda$ is the smallest root of $f_{4}(x ; p, q)=0$, i.e. $f_{4}(\lambda ; p, q)=0$, then $f_{4}(\lambda ; p+1, q-1)<0$, this implies $\lambda_{\text {min }}\left(G(p, q)^{c}\right)>\lambda_{\text {min }}\left(G(p+1, q-1)^{c}\right)$. So $\lambda_{\min }\left(G(2, n-4)^{c}\right)>\lambda_{\text {min }}\left(G(3, n-5)^{c}\right)>\cdots>$ $\lambda_{\min }\left(G(\lceil n / 2\rceil-1,\lfloor n / 2\rfloor-1)^{c}\right)$.

Next we will compare $\lambda_{\min }\left(G(n-3,1)^{c}\right)$ and $\lambda_{\min }\left(G(n-4,2)^{c}\right)$.

When $n \geq 21$ and $x<-\sqrt{37}$, we have

$$
\begin{aligned}
& (-x)^{2} f_{3}(x)-f_{4}(x ; n-4,2) \\
= & (-n+5) x^{5}+(-4 n+20) x^{4}+(2 n-10) x^{3} \\
& +(4 n-20) x^{2}-(n-5) x \\
> & (-n+5) x^{5}+(-4 n+20) x^{4}+(2 n-10) x^{3} \\
> & -x^{3}(n-5)\left(x^{2}+4 x-2\right) \\
> & 0 .
\end{aligned}
$$

Since $\lambda_{\min }\left(G(n-3,1)^{c}\right)<-\sqrt{2 n-5}<-\sqrt{37}$, then

$$
\lambda_{\min }\left(G(n-4,2)^{c}<\lambda_{\min }\left(G(n-3,1)^{c}\right) .\right.
$$

Then combining with Cases 1,2,3,4, the result follows.

\section{Main Results}

Theorem 3.1 Let $G$ be a connected graph of order $n(n \geq 21)$, which has pendent paths, then

$$
\lambda_{\text {min }}\left(G^{c}\right) \geq \lambda_{\min }\left(G(\lceil n / 2\rceil-1,\lfloor n / 2\rfloor-1)^{c}\right),
$$

with equality when and only when $G=G(\lceil n / 2\rceil-$ $1,\lfloor n / 2\rfloor-1)$.

Proof: Because of $K_{2} \subset G^{c}, \lambda_{\min }\left(K_{2}\right)=-1$. By Lemma 2.1, there is $\lambda_{\min }\left(G^{c}\right) \leq-1$. Let $\mathbf{x}$ be a first eigenvector of $G$. Denote $V^{+}=\left\{v: x_{v} \geq 0\right\}$ and $V^{-}=\left\{v: x_{v}<0\right\}$. By equation (2.2), we know that $\left|V^{+}\right| \geq 1,\left|V^{-}\right| \geq 1$. Let $\left|V^{+}\right|=m,\left|V^{-}\right|=t$, we have $m, t \geq 1$ and $m+t=n$, where $m, t$ are positive integers.

Denote $G[U]$ a subgraph of $G$, which induced on $U \subseteq V(G) ; E\left(V^{+}, V^{-}\right)$the set edges of $G$, every edge in which joins one vertex of $V^{+}$and one ver) tex of $V^{-}$. Since $G$ is a connected graph, there is at least one edge connecting $G\left[V^{+}\right]$and $G\left[V^{-}\right]$. By equation (2.1), in order to make $\mathbf{x}^{T} A(G) \mathbf{x}$ as large as possible, $E(G)$ must satisfy the following:

(i) $\left|E\left(V^{+}, V^{-}\right)\right|$should be as small as possible;

(ii) $\left|E\left(G\left[V^{+}\right]\right)\right|,\left|E\left(G\left[V^{-}\right]\right)\right|$should be as large as possible;

(iii) If $u v \in E\left(V^{+}, V^{-}\right),\left|x_{u}\right|$ and $\left|x_{v}\right|$ should be as small as possible;

(iv) If $u v \in E\left(G\left[V^{+}\right]\right)$or $u v \in E\left(G\left[V^{-}\right]\right),\left|x_{u}\right|$ and $\left|x_{v}\right|$ should be as large as possible.

By above discussions, the structure of $G$, and equations (2.3),(2.4), we get the following results:

(1) When $m=1$ or $t=1, \lambda_{\min }\left(G^{c}\right)=$ $\mathbf{x}^{T} A\left(G^{c}\right) \mathbf{x} \geq \mathbf{x}^{T} A\left(G(0, n-2)^{c}\right) \mathbf{x} \geq \lambda_{\min }(G(0, n-$ $\left.2)^{c}\right)$.

(2) When $m=2$ or $t=2, \lambda_{\min }\left(G^{c}\right)=$ $\mathbf{x}^{T} A\left(G^{c}\right) \mathbf{x} \geq \mathbf{x}^{T} A\left(G(0, n-2)^{c}\right) \mathbf{x} \geq \lambda_{\min }(G(0, n-$ $\left.2)^{c}\right)$.

(3) When $m=3$ or $t=3, \lambda_{\min }\left(G^{c}\right)=$ $\mathbf{x}^{T} A\left(G^{c}\right) \mathbf{x} \geq \mathbf{x}^{T} A\left(G(n-3,1)^{c}\right) \mathbf{x} \geq \lambda_{\min }(G(n-$ 
$\left.3,1)^{c}\right)$. or $\lambda_{\min }\left(G^{c}\right)=\mathbf{x}^{T} A\left(G^{c}\right) \mathbf{x} \geq \mathbf{x}^{T} A(G(3, n-$ $\left.5)^{c}\right) \mathbf{x} \geq \lambda_{\min }\left(G(3, n-5)^{c}\right)$.

(4) When $m, t \geq 4$, let $G_{3}=G(m, t-$ 2) or $G(t, m-2), \quad \lambda_{\min }\left(G^{c}\right)=\mathbf{x}^{T} A\left(G^{c}\right) \mathbf{x} \geq$ $\mathbf{x}^{T} A\left(G_{3}^{c}\right) \mathbf{x} \geq \lambda_{\min }\left(G_{3}^{c}\right)$.

$\operatorname{By}(1)(2)$ (3) (4) and Lemma 2.2, the conclusion is established.

Corollary Let $G_{n}$ be the set of the graphs of order $n(n \geq 21)$, whose complements are connected and have pendent paths. If $G \in G_{n}$, then

$$
\lambda_{\min }(G) \geq \lambda_{\min }\left(G(\lceil n / 2\rceil-1,\lfloor n / 2\rfloor-1)^{c}\right),
$$

with equality when and only when $G=G((\lceil n / 2\rceil-$ $\left.1,\lfloor n / 2\rfloor-1)^{c}\right)$.

\section{Conclusion}

Let $G_{n}$ be the set of the graphs of order $n$, whose complements are connected and have pendent paths. This paper investigates the least eigenvalue of the graphs and characterizes the unique graph which has the minimum least eigenvalue in $G_{n}$. Because relative to the results about spectral radius, there are few one on the least eigenvalue, and the method in this paper which studies the eigenvalue of the graphs from structure of their complements is a relatively new method, this paper is meaningful.

\section{Acknowledgments}

This work is jointly supported by the National Natural Science Foundation of China under Grant nos. 11071001, 11072059, and 61272530, the Natural Science Foundation of Jiangsu Province of China under Grant no. BK2012741, the Natural Science Foundation of Department of Education of Anhui Province nos. KJ2015ZD27, KJ2017A362.

\section{References}

[1] F. Bell, D. Cvetković, P. Rowlinson, S. Simić, Graph for which the least eigenvalues is minimal, I. Linear Algebra Appl., 429, 2008, 234-241.

[2] F. Bell, D. Cvetković, P. Rowlinson, S. Simić, Graph for which the least eigenvalues is minimal, II. Linear Algebra Appl., 429, 2008, 2168-2176.
[3] D. Cardoso, D. Cvetković, P. Rowlinson, S. Simić. A sharp lower bound for the least eigenvalue of the signless Laplacian of a non-bipartite graph, Linear Algebra Appl., 429, 2008, 2770-2780.

[4] Y. Fan, Y. Wang, Y. Gao, Minimizing the least eigenvalues of unicyclic graphs with application to spectralspread, Linear Algebra Appl., 429, 2008, 577-588.

[5] Y. Fan, F. Zhang, Y. Wang, The least eigenvalue of the complements of tree, Linear Algebra Appl., 435, 2011, 2150-2155.

[6] W. Haemers, Interlacing eigenvalues and graphs, Linear Algebra Appl., 227-228, 1995, 593-616.

[7] S. Li, S. Wang, The least eigenvalue of the signless Laplacian of the complements of trees, Linear Algebra Appl., 436, 2012, 2398-2405.

[8] R. Liu, M. Zhai, J. Shu Th,e least eigenvalues of unicyclic graph with $\mathrm{n}$ vertices and $\mathrm{k}$ pendant vertices. Linear Algebra Appl., 431, 2009, 657-665.

[9] M. Petrović, B. Borovićanin, T. Aleksić, Bicyclic graphs for which the least eigenvalue is minimum. Linear Algebra Appl., 430, 2009, 1328-1335.

[10] Y. Tan, Y. Fan, The vertex(edge) independence number, vertex(edge) cover number and the least eigenvalue of a graph, Linear Algebra Appl., 433, 2010, 790-795.

[11] Y. Wang, Y. Fan, The least eigenvalue of a graph with cut vertices. Linear Algebra Appl., 433, 2010, 19-27.

[12] Y. Wang, Y. Fan, X. Li, et al. The least eigenvalue of graphs whose complements are unicyclic, Discussiones Mathematicae Graph Theory, 35(2), 2013, 1375-1379.

[13] M. Ye, Y. Fan, D. Liang. The least eigenvalue of graphs with given connectivity. Linear Algebra Appl., 430, 2009, 1375-1379.

[14] G. Yu, Y. Fan, Y. Wang, Quadratic forms on graphs with application to minimizing the least eigenvalue of signless Laplacian over bicyclic graphs, Electronic J. Linear Algebra, 27, 2014, 213-236.

[15] G. Yu, Y. Fan. The least eigenvalue of graphs. Math. Res. Expo., 32(6), 2012, 659-665.

[16] G.Yu, Y.Fan, The least eigenvalue of graphs whose complements are 2-vertex or 2-edge connected, Operations Research Transactions, 17(2), 2013, 81-88. 


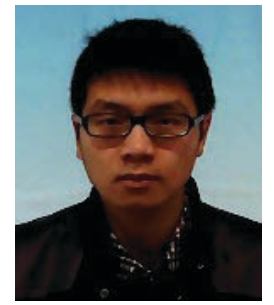

Chen Wang received the Engineer diploma in the Automation from Shenyang Institute of Technology, China in 2014. Currently, he is doing his master's degree at Guizhou University. His research interests include optimal control, econometrics and graph theory.

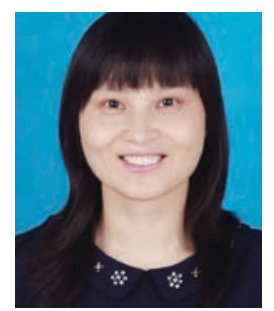

Guidong Yu is a Professor of Anqing Normal University. She received the Research Master Degree in Graph Theory from Anhui Normal University in 2006, and the Ph.D. Degree in Algebraic Graph Theory from the Anhui University in 2012. Her research interests include graph theory, algebraic graph theory and combinatorial matrix theory.

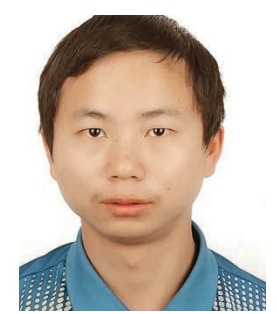

Wei Sun received the bachelor of science in the Mathematics from Huainan Normal University, China in 2014. Currently, he is doing his master's degree at Anqing Normal University. His research interests include algebraic graph theory and combinatorial matrix theory.

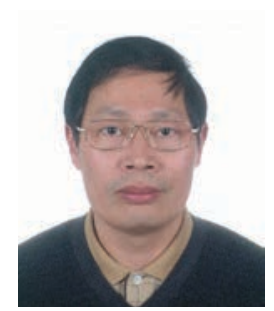

Jinde Cao is an Endowed Chair Professor, the Dean of School of Mathematics and the Director of the Research Center for Complex Systems and Network Sciences at Southeast University. From March 1989 to May 2000, he was with the Yunnan University. In May 2000, he joined the School of Mathematics, Southeast University, Nanjing, China. From July 2001 to June 2002, he was a Postdoctoral Research Fellow at Chinese University of Hong Kong, Hong Kong. Professor Cao was an Associate Editor of the IEEE Transactions on Neural Networks, and Neurocomputing. He is an Associate Editor of the IEEE Transactions on Cybernetics, IEEE Transactions on Cognitive and Developmental Systems, Journal of the Franklin Institute, Mathematics, and Computers in Simulation, Cognitive Neurodynamics, and Neural Networks. He is a Fellow of IEEE, a Member of the Academy of Europe, a Member of European Academy of Sciences and Arts, and a Foreign Fellow of Pakistan Academy of Sciences. He has been named as Highly-Cited Researcher in Engineering, Computer Science, and Mathematics by Thomson Reuters/Clarivate Analytics. He received the National Innovation Award of China (2017). 